\title{
EL ESTUdIO PSICOLÓgICO COMPARADO DE LA PERCEPCIÓN DE LOS CONTORNOS DE ENTONACIÓN DECLARATIVO E INTERROGATIVO*
}

\section{THE COMPARATIVE PSYCHOLOGICAL STUDY OF THE PERCEPTION OF INTONATION CONTOURS FOR DECLARATIVE AND INTERROGATIVE SENTENCES}

\author{
LEONARDo Barón BiRCHENALL* \\ *Trabajo realizado en el marco de la investigación titulada Discriminación de los contornos de entonación \\ declarativo e interrogativo en ratas, financiada por la Fundación Universitaria Los Libertadores y apoyada \\ por el Grupo de Estudios en Ciencias del Comportamiento. \\ **Psicólogo y Magíster en Psicología Cognitiva. Investigador y Docente de la Fundación \\ Universitaria Los Libertadores y Miembro del Grupo de Estudios en Ciencias del Comportamiento. \\ E-Mail: laescaladesol@gmail.com - Diagonal 61c - 22a 17 Bogotá, Colombia.
}

REsUmEN

Si bien existen numerosas investigaciones sobre la percepción del lenguaje en recién nacidos, la percepción de la entonación en adultos y la $P s i-$ cología Comparada del lenguaje, son muy escasos los estudios en cuanto a la percepción de los contornos de entonación declarativo e interrogativo con bebés, y los estudios sobre este mismo tema en modelos animales son al parecer inexistentes. En este artículo se plantean los fundamentos del estudio comparado de la percepción de dichos contornos de entonación en bebés y en animales. En primer término se discute acerca de la composición del lenguaje y el enfoque de estudio psicolingüístico comparado. A continuación se tratan los temas de la isocronía y de los contornos de entonación declarativo e interrogativo, argumentando que en ambos casos existe un alto grado de universalidad. Finalmente se analizan los alcances del tipo de estudio propuesto, indicando su viabilidad y pertinencia.

Palabras clave: Percepción del habla; Entonación declarativa e interrogativa; Psicología Comparada; Universales lingüísticos; Modelos animales.

\section{ABSTRACT}

One of today's most influential theories of language development and acquisition assumes that the human faculty of language is made-up of three components: syntax, phonology, and semantics. Of these components, the syntactic one may be exclusive of humans, while some specific aspects of the semantic and phonological ones may be not. One implication of this division of the language faculty is that aspects of the semantic and phonological systems could also be found in non-human animals, with their function not necessarily being related to communication. Even though not all the scientific community accepts such explanation of the components of the language faculty, diverse academic groups that do so, have been doing research with humans, non-human primates, rodents, birds, and even with cats, for the last 25 years or so. Usually, the purpose of this type of studies has been to determine what human capacities related to speech have emerged from different precursor systems that were already present in ancestral species, and would have been inherited by men, which components of language are specifically human, which are specifically linguistic, and which ones could be domain-general mechanisms 
or have served originally for other purposes. Several of these studies imply the existence of a universal division of languages according to rhythmic considerations, known as isochrony. According to this concept, each natural language possesses stable units of constant duration that occur regularly, making it sound in a particular way. But isochrony is not the only universal language feature that can constitute a valid variable in this sort of study. Some characteristics of intonation may also be. Specifically the declarative and interrogative intonation contours, which have been considered a universal phenomenon present in comparable ways in all natural languages. In this regard, though in the different languages of the world the distinction between declaratives and interrogatives can be marked by means of morphsyntactic modifications and intonation, not be marked by intonation, or be only marked by intonation, it is believed that there is a universal tendency to use rising intonation contours to indicate questions (especially in the case of unemphatic yes / no questions) and falling intonation contours to indicate statements. This phenomenon would be related to certain non-linguistic universal codes shared by the human broad phylum, and derived from biologically determined relations. However, while there are numerous investigations in the newborns' and infants' general speech perception processes, in perception of intonation of declarative and interrogative sentences in adults, and in Comparative Psychology of language, studies with infants and newborns in terms of the discrimination of questions and statements are insufficient, and studies on this topic on animal models are, as far as we know, non-existent. In this paper we theoretically validate the comparative study of the perception of intonation contours for questions and statements in infants and in nonhuman animals. We conclude that is feasible and relevant to increase the study of the infants' perception of declarative and interrogative intonation contours, with meaningful variations with respect to the experiments already done, such as the length of the utterances, the type of speech register, the words order, the use of grammatical auxiliaries, the ages of the participants, and the experimental methods. Moreover, it is also desirable to make that same kind of perceptual studies of intonation on animal models, such as rats, birds and monkeys. Pursuing these ob- jectives would make it possible to deepen the knowledge about the language components that are unique for humans and those that are shared with other species. It also could shed some light on the form-function relationships in the phonological component of the language, and about the linguistic universals and precursors.

Key words: Speech perception; Declarative and interrogative intonation; Comparative Psychology; Linguistic universals; Animal models.

\section{INTRODUCCIÓN}

El objetivo del estudio que se informa fue plantear una vía para explorar aspectos del lenguaje que pueden ser privativos de los humanos o ser compartidos con otras especies; específicamente el funcionamiento de la percepción de los contornos de entonación declarativo e interrogativo.

Para este fin, debe considerarse que actualmente existen numerosas investigaciones sobre la percepción del lenguaje en recién nacidos, la percepción de la entonación en adultos y en la Psicología Comparada del lenguaje, pero los trabajos que abordan la percepción de los contornos de entonación declarativo e interrogativo por parte de bebés se reducen básicamente a cuatro, y los estudios sobre este mismo tema en modelos animales son al parecer inexistentes.

En este artículo se argumenta que aumentar el número de estudios de la percepción de preguntas y declaraciones en recién nacidos, realizando modificaciones clave respecto a los trabajos existentes, resulta viable y pertinente; así como resulta deseable realizar el mismo tipo de investigación sobre percepción de la entonación en modelos animales.

\section{COMPOSICIÓN DEL LENGUAJE Y ESTUDIO PSICOLIN- GÜÍSTICO COMPARADO}

En un sentido general puede entenderse que la facultad humana del lenguaje está constituida por un componente sintáctico, 
uno fonológico y uno semántico. De estos tres componentes es posible que el sintáctico sea privativo de los humanos, mientras que aspectos de los componentes semántico y fonológico no lo sean (Berwick, Friederici, Chomsky \& Bolhuis, 2013; Di Sciullo et al., 2010; Hauser, Chomsky \& Fitch, 2002). Desde esta perspectiva la facultad del lenguaje se divide en facultad del lenguaje en sentido amplio (FLB) y facultad del lenguaje en sentido restringido (FLN). La FLB abarcaría el sistema sensorial-motor, el sistema conceptual-intencional y la FLN, pero no otros sistemas, como la memoria. La FLN correspondería a un mecanismo abstracto computacional, propio de los humanos y caracterizado por la recursividad; la cual es una propiedad que permite producir una variedad potencialmente infinita de expresiones a partir de una serie finita de elementos (cf., Chomsky, 1997).

Siguiendo esta lógica, aspectos de los sistemas sensorial-motor y conceptual-intencional podrían hallarse también en animales no humanos sin que necesariamente su función estuviera relacionada con la comunicación. En el caso de los humanos los sistemas sensorial-motor y conceptual-intencional habrían aparecido con anterioridad a la FLN (filogenéticamente hablando). La aparición de la FLN hace unos 75000 años en un individuo (en el este de África), debido a una mutación menor (un ligero recableado del cerebro), habría conectado los sistemas sensorialmotor y conceptual-intencional entre sí, funcionando como una suerte de puente y posibilitando así que se generara la facultad del lenguaje tal como se la conoce en la actualidad (Berwick \& Chomsky, 2011; Chomsky, 2010, 2011).

Sin embargo, esta forma de explicar la composición y la aparición filogenética del lenguaje no es aceptada por toda la comunidad científica. Por ejemplo, Pinker y Jackendoff (2005) dudan que sea la recursividad el componente específicamente humano del lenguaje, pues este planteamiento ignora aspectos lingüísticos que no son recursivos y que sí podrían resultar siendo privativos de los humanos, como aspectos de la fonología y la morfología, el caso, la concordancia (agreement) y ciertas propiedades de las palabras. Además, estiman que existe por lo menos un gen relacionado con el habla que fue seleccionado evolutivamente en el linaje humano y que no está relacionado con la recursividad $\left(\mathrm{el} \mathrm{FOXP2}{ }^{1}\right)$. Sobre los aspectos del lenguaje compartidos con otras especies, en el caso del componente sensorial-motor, aducen que tal posibilidad es inconsistente con la anatomía y el control neural del tracto vocal humano y que además es incompatible con evidencia empírica que indica que la percepción del habla no puede ser reducida a la audición de los primates y que el aprendizaje de palabras no puede ser reducido al aprendizaje de datos.

Diversos grupos de académicos que están de acuerdo con la existencia de componentes del lenguaje compartidos en alguna medida con otras especies, están realizando desde hace unos 25 años numerosas investigaciones con humanos, monos, roedores, aves y hasta con gatos. La finalidad de estos estudios ha sido en general determinar qué capacidades relacionadas con el habla han sido heredadas por el hombre a partir de distintos sistemas precursores que ya estaban presentes en especies ancestrales -el punto de vista

1 En el año 2001 se descubrió que una mutación del gen FOXP2 está directamente relacionada con un subtipo del specific language impairment en varios miembros de una familia inglesa. Aunque hay polémica sobre lo que esto significa, en general se entiende que los trastornos generados por la mutación del gen son de orden lingüístico. De cualquier forma, explica Longa (2006), a partir de lo encontrado no se puede establecer una relación directa entre el gen no mutado y la facultad del lenguaje (entre otras cosas, porque los genes proveen la información para sintetizar las proteínas a partir de los aminoácidos, pero no proveen patrones de comportamiento). Además, no hay una relación directa entre un gen y un rasgo; por el contrario parece ser que las interacciones entre cadenas de genes se relacionan con uno u otro rasgo. En conclusión, el gen FOXP2 está relacionado con el lenguaje, pero no necesariamente es el gen del lenguaje (de la sintaxis o de la articulación). 
según el cual este fenómeno ocurre se conoce como hipótesis auditiva-, qué componentes del lenguaje son específicamente humanos y cuáles podrían ser mecanismos de dominio general o haber servido originalmente para otros propósitos (Gervain \& Mehler, 2010; Ohms, Gill, Heijningen, Beckers \& ten Cate, 2010; Sinnott \& Gilmore, 2004).

No obstante, tampoco existe consenso en la comunidad académica sobre la validez de este tipo de estudios comparados del habla, pues se afirma que encontrar equivalencias conductuales como respuesta a estímulos lingüísticos, o semejanzas de tipo estructural entre los hombres y otros animales, no permite afirmar que existan capacidades compartidas entre las especies estudiadas. Por lo tanto, no bastaría solamente con demostrar isomorfismos conductuales entre especies, referidos por ejemplo a la percepción del habla, sino que además deberían encontrarse mecanismos biológicos y organizaciones funcionales comunes relacionados con la capacidad lingüística (Trout, 2001, 2003).

Algunos de los investigadores que desestiman el estudio comparado del habla sostienen además que el lenguaje es especial (teoría conocida como $\mathrm{SiS}$, por Speech is Special), argumentando que la capacidad lingüística está fundamentada en la particular biología de los humanos y es específica de ellos; de modo que las formas particulares de percepción y producción del lenguaje serían privativas de los humanos: adaptaciones singulares al ambiente que implicarían mecanismos y leyes especiales. En este sentido la percepción del habla respondería a un tipo de adaptación biológica relativa específicamente a fenómenos lingüísticos y no a fenómenos acústicos generales (Pinker \& Jackendoff, 2005).

Con respecto a la SiS, Liberman (1982) propuso que existen procesos especializados de percepción fonética cuyo propósito es responder a los patrones acústicos que resultan de la forma en que los movimientos articulatorios son regulados. La percepción de los segmentos fonológicos dependería entonces de procesos psicoacústicos especializados que sirven a una función netamente lingüís- tica y que serían parte de una especialización mayor con respecto a aspectos de producción, comprensión y adquisición del lenguaje.

Para apoyar esta forma de comprender el lenguaje (SiS) se refiere evidencia empírica relativa al fenómeno de restauración de fonemas (cuando en condiciones experimentales se presenta una palabra sin uno de sus fonemas, pero los sujetos reportan que lo han escuchado), a la percepción doble (cuando en condiciones experimentales se presenta una especie de chirrido por un oído y por el otro un fonema incompleto y los sujetos refieren la percepción de ambos estímulos en forma integral), a la ventaja del oído derecho (más precisión y rapidez en la identificación de fonemas en el habla mediante este oído) y a la lateralización del cerebro (que se puede constatar mediante el paradigma de escucha dicótica) (Heimbauer, 2012).

Al margen de la polémica sobre la composición del lenguaje y sus elementos compartidos entre especies, la investigación lingüística comparada ha avanzado significativamente en las últimas décadas, arrojando datos que han permitido ir aclarando paulatinamente qué es y cómo funciona la facultad humana del lenguaje, así como sus relaciones con los sistemas de comunicación animal. Para alcanzar este fin, los resultados de los estudios en humanos adultos y recién nacidos son contrastados regularmente con información obtenida del trabajo con roedores, aves y monos. Los aspectos respecto a los cuales se realizan comparaciones abarcan la discriminación de fonemas, sílabas, palabras y oraciones, el aprendizaje de patrones gramaticales simples y de palabras, el rastreo de regularidades distributivas de elementos (probabilidades transicionales y frecuencia de ocurrencia conjunta) y la extracción y generalización de reglas algebraicas en la señal del habla (entre otros: Brainard \& Doupe, 2002; Elgier \& Bentosela, 2009; Endress, Cahill, Block, Watumull \& Hauser, 2009; Ghazanfar \& Maier, 2009; Jakovcevic \& Bentosela, 2012; Murphy, Mondragón \& Murphy, 2008; Newport, Hauser, Spaepen \& Aslin, 2004; Ohms, Escudero, Lammers \& ten Cate, 2012; 
Pilley \& Reid, 2011; Reed et al., 2003; Saffran et al., 2008; Sinnott \& Gilmore, 2004; Toro \& Trobalon, 2005; para una revisión general ver Barón, 2014).

\section{LA ISOCRONÍA Y LOS CONTORNOS DE ENTONACIÓN DECLARATIVO E INTERROGATIVO: VARIABLES DE ES- TUDIO PSICOLINGÜÍSTICO DE CARÁCTER UNIVERSAL}

Algunos estudios comparados del lenguaje suponen la existencia de una división general de las lenguas según consideraciones rítmicas, conocida como isocronía. De acuerdo con el concepto de isocronía cada lengua posee unidades estables de duración constante que se repiten regularmente. Estas unidades estables de duración producen diferencias en el modo en que son percibidas las distintas lenguas del mundo (Ramus, 1999). Tradicionalmente se han considerado tres tipos de isocronía (Gervain \& Mehler, 2010; Toledo, 2009):

1.- Lenguas de compás silábico: las sílabas son el elemento rítmico esencial, pues tienen en general duraciones más o menos similares (por lo menos se perciben así). En esta categoría se encuentra el español, el francés, el italiano (en general las lenguas romances), el hindi, el mandarín y el griego. Los idiomas de este grupo presentan una suerte de ritmo de ametralladora.

2.- Lenguas de compás acentual: el acento es el elemento rítmico fundamental; así, aunque las sílabas pueden tener duraciones diferentes se percibe una cantidad constante de tiempo en promedio entre las sílabas acentuadas. En este grupo se encuentra el inglés, el alemán, el holandés (en general las lenguas germánicas), el árabe y el ruso. En ocasiones se les llama lenguas de ritmo de código Morse.

3.- Lenguas de compás de mora: la mora es el elemento rítmico esencial, de tal forma que cada una de ellas tiene duraciones equivalentes (la mora es una medida de la sílaba, las sílabas cortas tienen una mora y las largas dos - Gervain \& Mehler, 2010). Ejemplo típico de estas lenguas es el japonés.

En general, aunque estas categorías rítmicas son aceptadas y usadas como marco de referencia y comparación, los estudios sobre la señal del habla indican que la clasificación de la isocronía es falsa, pues no se ha encontrado que las unidades relevantes de tiempo (sílaba, acento y mora) duren lo mismo en las lenguas en las que deberían tener igual duración; además, se han reportado varios idiomas que podrían pertenecer a dos categorías a la vez (Mehler, Christophe \& Ramus, 2000; Peña, Pittaluga \& Mehler, 2010).

Sin embargo, Ramus, Nespor y Mehler (1999), a partir del estudio de ocho idiomas, en cuanto a su constitución y a la forma en que las personas los perciben, concluyen que la proporción de intervalos vocálicos (tiempo entre el final de una vocal y el comienzo de la siguiente) y la variabilidad de los intervalos entre consonantes son congruentes con la clasificación tradicional de la isocronía. Consideran estos autores que el ritmo, concebido como la alteración de la duración de vocales y consonantes, es una propiedad esencial que permite diferenciar los idiomas y adquirir la lengua materna. Las conclusiones en general de su trabajo apoyan la existencia y relevancia de las clases rítmicas en el orden de la percepción. Evidencia adicional (no concluyente) a favor de la realidad de la isocronía se encuentra en el trabajo de Ramus, Dupoux y Mehler (2003).

Por lo tanto, si la isocronía es una característica inherente a las lenguas naturales, o al menos a la percepción que las personas tienen de ellas, buscar respuestas conductuales equivalentes o disímiles en distintos animales ante la señal del habla, controlando la variable de la isocronía, es una línea de estudio viable en el marco de la Psicología Comparada del lenguaje.

Pero la isocronía no es la única característica universal del lenguaje que puede constituir una variable válida para el estudio psicolingüístico comparado: algunas caracterís- 
ticas de la entonación también pueden serlo. Específicamente los contornos de entonación declarativo e interrogativo, los cuales han sido considerados también un fenómeno de carácter universal presente en formas equivalentes en todas las lenguas naturales.

Ahora bien, aunque no hay un consenso académico sobre lo que es la entonación, se considera usualmente que al igual que el ritmo, es una de las características suprasegmentales del habla, en ocasiones referidas como prosodia (Lehiste, 1970; Vaissière, 2005); es usual que los términos prosodia y entonación se confundan y se usen de modo intercambiable en la literatura especializada (cf., Hirst \& Di Cristo, 1998). Se entiende que la entonación es una especie de melodía ejecutada por las variaciones de la voz: la melodía con que se canta o entona lo que se dice. Esta melodía acompaña la secuencia de fonemas de los enunciados, pudiendo reflejar diferencias de sentido, de intención, de emoción y de origen. La secuencia de tonos que conforman la entonación de un enunciado se conoce como contorno o curva de entonación (Alarcos, 2000).

La transmisión de información mediante la entonación se realiza a partir de las variaciones de la frecuencia fundamental de la voz (o formante cero: $F 0$ ), producidas por el ciclo vibratorio de las cuerdas vocales. La frecuencia fundamental tiene como correlato perceptual la altura tonal o pitch. Manipulando la rigidez y la longitud de las cuerdas vocales, elevando o bajando la laringe y cambiando la presión subglótica, los seres humanos controlan las distintas características del $F 0$ (Vaissière, 2005).

En cuanto a la función de la entonación en el reino animal, se sabe que en general los mamíferos pueden aprender relaciones entre señales acústicas sutiles y eventos (incluidas necesidades físicas y estados emocionales), lo cual les permite transmitir información de diversos tipos por medio de variaciones prosódicas. Así, el hombre y los demás mamíferos usan variaciones rítmicas y de tonalidad en sus vocalizaciones para distintos propósitos, como la comunicación entre madre e hijo, las advertencias a los contendientes, asun- tos de socialización y expresión de emociones y necesidades en general (Lieberman, 1996).

Con respecto al lenguaje humano en particular, se sabe que toda lengua tiene entonación y que muchas de las funciones lingüísticas y paralingüísticas de la entonación parecen ser compartidas por lenguajes de muy distintos orígenes (Hirst \& Di Cristo, 1998). Además, de acuerdo con Vaissière (2005), a pesar de las diferencias obtenidas en experimentos con hablantes nativos de diversas lenguas sobre la percepción de la entonación, la literatura referida a una gran cantidad de idiomas (relacionados y no relacionados entre sí) señala la existencia de varias tendencias universales en la relación entre forma y significado.

Entre estas tendencias resalta el uso de caídas en la entonación (disminución del $F 0$ ) relacionadas con categorías de significados abstractos cerrados: asertivos, de refuerzo, declarativos, dogmáticos, finales y propicios. Y el uso de aumentos de la entonación (aumento del $F 0$ ) relacionados con categorías de significados abstractos abiertos: no asertivos, limitantes, interrogativos, de continuidad, poco propicios, declarativos con reservas y conciliadores (Cruttenden, 1981).

El caso particular de la entonación relativa de los enunciados interrogativos y declarativos es polémico, pues si bien algunos autores encuentran que no hay un patrón específico de entonación sistemáticamente asociado con los enunciados interrogativos, según afirman Hirst y Di Cristo (1998), refiriéndose a trabajos referidos al holandés, al griego y al finlandés, en un gran número de otros idiomas se encuentra que diferencias en los contornos de entonación de los enunciados se corresponden regularmente con diferencias en sus modos (entendidos en sentido de interrogativo, declarativo, exclamativo, dubitativo, etc.). Al respecto:

"It is commonly maintained that a distinction between declarative and interrogative modes is one of the most universal characteristics of intonation systems [...] It has often been noted, for example, that in a vast majority of languages some sort of raised pitch 
(final or non-final) can be used in contrast with lower pitch to indicate that an utterance is intended as a question rather than as a statement" (Hirst \& Di Cristo, 1998, pp. 1 y 24).

Así, aunque en las distintas lenguas del mundo la distinción entre enunciados declarativos e interrogativos puede estar marcada por modificaciones morfosintácticas y de entonación, no estar marcada por la entonación, o estar marcada solo por la entonación (Frota, Butler \& Vigário, 2013), se cree que existe una tendencia universal a usar $F 0$ (s) altos y/o elevaciones del $F 0$ para marcar interrogaciones (sobre todo en el caso de las preguntas sin énfasis que se responden con sí o no), y $F 0(\mathrm{~s})$ bajos y/o caídas del $F 0$ para marcar declaraciones (Ohala, 1984; Soderstrom, Ko \& Nevzorova, 2011; Yang \& Liang, 2012²).

De cualquier manera, existen diversos tipos de interrogaciones. En el caso de las preguntas sin énfasis que se responden con sí o no se describe comúnmente un aumento en el tono del enunciado, en forma total o parcial, independientemente de un aumento final (no obstante siempre existen preguntas sin elevación del $F 0$ y elevación del $F 0$ sin pregunta) (Geffen \& Mintz, 2011). Sobre este tipo de preguntas afirman Hirst y Di Cristo (1998, p. 25):

"By far the most commonly described characteristic of questions is a high final pitch. This corresponds to the intuition that questions have rising intonation whereas de-

2 En el estudio de Yang y Liang (2012), pacientes con daño cerebral en el hemisferio derecho emitieron contornos de entonación descendentes tanto en declaraciones como en interrogaciones, indicando una dificultad para generar la entonación de las preguntas. Sostienen estos autores que el hemisferio izquierdo está relacionado con el procesamiento de características segmentarias del habla, como el acento léxico, mientras que el hemisferio derecho está relacionado con el procesamiento de características suprasegmentarias, como la entonación. claratives have falling intonation. Bolinger (1978b) reported that about $70 \%$ of a sample of nearly 250 languages were said to use a rising terminal to signal questions and that the remaining 30\% used a higher over-all pitch for questions than for non-questions."

El caso de las preguntas con palabras interrogativas, o palabras qu, es distinto, pues en diversas lenguas (como el inglés, el español, el ruso y el griego) su entonación se describe como más similar a una declaración que a una pregunta que se responde con sí o no. En las preguntas que exigen una repetición de todo o parte de un enunciado, o en las que se repite, por ejemplo:

- Emi vino la semana pasada

- ¿Cuándo?

- La semana pasada;

- Juan salió por la noche

- ¿Que Juan salió por la noche?,

parece producirse una entonación en aumento particularmente enfatizada en muchas lenguas. Para las preguntas que se valen de una declaración con un fragmento interrogativo final (por ej.: - Fuiste al parque, ¿no es así?) se describen entonaciones variadas. Las oraciones sin terminar, al igual que las preguntas que se responden con sí o no, son comúnmente pronunciadas en distintos idiomas con un aumento final en la entonación (Geffen \& Mintz, 2011; Hirst \& Di Cristo, 1998).

No obstante, en idiomas como el español castellano la entonación de los declarativos y de los interrogativos (absolutos) difiere en diversas características, como la altura del pico del $F 0$, la presencia o ausencia de acentos de tono y la dirección del movimiento final del $F 0$ (Face, 2005). Ahora bien, en un estudio realizado por este mismo autor (Face, 2005) se encontró que se puede diferenciar entre un enunciado declarativo y uno interrogativo absoluto (en español castellano) con aproximadamente un $95 \%$ de efectividad, solamente a partir de la percepción de la primera sílaba postónica del enunciado, la cual contiene el primer pico del $F 0$ del con- 
torno de entonación. Sin embargo, refiere también Face, se ha encontrado que en holandés, en la diferenciación entre declarativos e interrogativos, la presencia o ausencia final de un aumento en la entonación del enunciado anula las conclusiones debidas al resto de información que se ha percibido con anterioridad.

Una posible explicación al fenómeno de aumento de entonación en las preguntas y descenso en las declaraciones ha sido propuesta por Ohala (1984), quien estima que las semejanzas existentes en los lenguajes respecto a contornos de entonación en declaraciones y en interrogaciones están estrechamente relacionadas con las semejanzas en diversas culturas con respecto a la entonación en la expresión vocal de actitudes y afectos, las semejanzas en diversas especies en el uso del $F 0$ para vocalizaciones amenazantes y no amenazantes, y el dimorfismo sexual en la anatomía del aparato fonador de los humanos $\mathrm{y}$ de algunos otros animales.

Todos estos fenómenos responderían a una asociación innata de las frecuencias acústicas altas con el significado primario de vocalizador pequeño, y con significados secundarios de subordinación, sumisión, no amenaza y deseos de buena voluntad de quien vocaliza. $\mathrm{Y}$ a una asociación equivalente de las frecuencias acústicas bajas con el significado primario de vocalizador grande y con significados secundarios de dominancia, agresividad y amenaza.

Esta asociación podría darse en virtud del llamado código de frecuencia, el cual se basa en el hecho de que pequeñas laringes producen notas más altas que laringes más grandes; lo cual lleva a que el tono más alto suene vulnerable y sumiso, mientras que el más bajo suene protector y dominante. De esta forma, los enunciados en tono alto, en particular los que terminan en tono alto, parecen sonar dependientes, atractivos e interrogantes; mientras que los enunciados en tono bajo, en especial los que terminan en tono bajo, parecen sonar autoritarios, poderosos y asertivos (Gussenhoven \& Chen, 2000).

En términos generales la existencia del código de frecuencia es aceptada por la co- munidad académica, estima Vaissière (2005), quien además enfatiza que el código no solo aplica a las expresiones del lenguaje humano, sino que también puede abarcar comunicaciones de otros animales. En este sentido, se sabe que los vertebrados terrestres no humanos usan sonidos similares en forma similar, de manera que la estructura de la señal permite al receptor predecir su función. Los sonidos de tono bajo se usan para mantener lejos a un sujeto (mostrándose amenazante) y los de tono alto para mantener la distancia (al evidenciar que no hay amenaza o indicar miedo), aproximarse a otro, aparearse o advertir a los pares de algún peligro (Morton, 1994).

Esta forma de simbolismo sonoro expresivo podría ser muy antigua en la escala evolutiva y haber estado presente incluso en los dinosaurios, heredándose de ellos a reptiles y aves (Morton, 1994). En reptiles y anfibios, la frecuencia de las vocalizaciones habría resultado en el reflejo directo del tamaño del animal debido a la cantidad de masa involucrada en la vibración (frecuencias más bajas, animal más grande; frecuencias más altas, animal más pequeño). En aves y mamíferos las frecuencias altas o bajas no reflejarían directamente diferencias de tamaño sino diferencias de motivación. En consecuencia, se cree que un principio fundamental de las vocalizaciones animales emitidas en contextos hostiles o amistosos es la transmisión de una impresión del tamaño del vocalizador (Vaissière, 2005).

En consecuencia, el código de frecuencia no existiría en la comunicación lingüística por ser parte del lenguaje en sí, sino por corresponder a una herencia biológica más amplia. De esta forma el ajuste de las estructuras del lenguaje a este código puede ser una forma de volver gramatical un fenómeno que no es lingüístico. Los hablantes sabrían utilizar estos códigos universales no lingüísticos debido a que son compartidos por nuestro amplio filo y son derivados de relaciones determinadas biológicamente; por lo tanto serían innatos (Gussenhoven \& Chen, 2000).

Por otra parte, Ladd (1981) identificó dos puntos de vista en cuanto a la relación entre la entonación y los significados que se expre- 
san mediante su uso: la hipótesis de tono nuclear y la hipótesis universal fuerte. Según esta última, la función lingüística de la entonación está determinada en forma innata y/o responde a estados fisiológicos naturales del hablante. De acuerdo con este punto de vista, los aumentos del tono señalan las preguntas y las caídas señalan las declaraciones, pues un tono alto o una elevación de tono al final de un enunciado indica que está incompleto o que no ha sido resuelto, mientras que un tono bajo o una caída de tono al final del enunciado indica que está completo o que ha sido resuelto. Ambos fenómenos se deberían al hecho de sostener o reducir la tensión muscular, dependiendo de la intención de continuar o detener la vocalización. Así, usar terminaciones de tono alto o elevaciones en la entonación de pregunta señala falta de resolución o no completitud en el orden del discurso, de manera que uno de los hablantes invita al otro a resolver la interacción (una forma es respondiendo).

Según la hipótesis de tono nuclear, por la cual opta Ladd (1981), la relación entre los contornos tonales y los significados es arbitraria y está determinada por la gramática de cada idioma. De acuerdo con este punto de vista existen tonos nucleares, como los de caída, caída-aumento, alto-aumento y bajoaumento, en el idioma inglés. Cada lengua tendría su propio inventario de tonos nucleares y el tono nuclear que se utilice para algo determinado en un idioma puede usarse para otra cosa en otro idioma.

En respuesta a la defensa de Ladd de la hipótesis de tono nuclear, señala Ohala (citado en Ladd, 1981) que al emitir un sonido de tono alto un animal trata ocasionalmente de imitar el sonido de tono alto de un animal joven de su misma especie con el fin de activar en el otro alguna clase de respuesta parental. El tono de las vocalizaciones de los animales jóvenes sería necesariamente alto pues al ser más pequeños tienen cuerdas vocales, o aletas de la siringe en el caso de las aves, de menor tamaño y que por lo tanto producen $F 0(\mathrm{~s})$ más altos. De esta forma, concluye Ohala, si tenemos esta clase de programación innata dentro de nosotros es natural que algo de ella se manifieste en nuestro uso lingüístico de la frecuencia fundamental.

Por otra parte, de acuerdo con Gussenhoven y Chen (2000) la explicación de la hipótesis universal fuerte sobre la relación entre la entonación y los significados que se expresan mediante su uso ha sido largamente documentada y cuenta con amplio apoyo empírico, incluido un estudio realizado por estos mismos autores con sujetos hablantes de idiomas en los que los enunciados interrogativos se expresan en formas distintas (entre los idiomas) en términos de su contorno de entonación (chino mandarín, holandés y húngaro). A estos hablantes se les presentaron pares de estímulos sonoros específicamente dispuestos en cuanto a sus características acús- ticas y se les pidió que juzgaran cuál de ellos parecía más una pregunta (los estímulos no pertenecían a ninguna lengua natural, aunque se les decía que pertenecían a una lengua exótica). En general fueron seleccionados los estímulos con el pico más alto, el pico más tardío y el mayor aumento final como los que indicaban una pregunta, independientemente de la lengua materna de quien juzgaba. Los autores del experimento concluyen que estos resultados reflejan un conocimiento innato no lingüístico del significado de la variación del tono, que responde específicamente al código de frecuencia.

Evidencia adicional arrojó el estudio de Yuan, Shih y Kochanski (2002), según el cual las diferencias entre la entonación declarativa e interrogativa en chino se deben a valores altos más fuertes en los tonos finales y a un contorno de entonación en general más alto, en las oraciones interrogativas. Estos resultados, sumados a los del estudio de Gussenhoven y Chen (2000) indican que las personas tienden a interpretar las oraciones con picos de entonación altos y/o con tonos finales altos como enunciados interrogativos independientemente de su lengua materna.

Las excepciones encontradas en distintos idiomas al uso de $F 0$ (s) altos y/o elevaciones del $F 0$ para señalar preguntas y $F 0(\mathrm{~s})$ bajos y/o caídas del $F 0$ para señalar declaraciones podrían explicarse en términos de contradicciones fortuitas entre un determinado lexicón 
de entonación (específico de cada lenguaje y compuesto por un grupo de melodías nucleares) y los significados compartidos por todas las lenguas (Gussenhoven \& Chen, 2000).

De cualquier manera, afirman Hirst y Di Cristo (1998) que las características particulares de un sistema de entonación dependen en gran medida no solo del lenguaje, sino también del dialecto, del estilo, del estado de ánimo y de la actitud de los hablantes. Además, si bien estos autores encuentran que en la mayoría de los 20 idiomas que se analizan en su libro, un tono final alto es una forma habitual de señalar que un enunciado es una pregunta, en otros idiomas, como el danés, el finlandés y el árabe, no lo es. Sin embargo, concluyen que algunas de las lenguas que no presentan un tono final alto como forma de marcar una interrogación, sí usan comúnmente un tono final ascendente en los enunciados incompletos.

Por su parte, estima Ohala (1984) que pese a las excepciones a la tendencia general en los lenguajes del mundo a usar $F 0(\mathrm{~s})$ altos y/o elevaciones del $F 0$ para indicar preguntas (sobre todo las que se responden con sí o no), y $F 0(\mathrm{~s})$ bajos y/o caídas del $F 0$ para indicar declaraciones, la alta incidencia del fenómeno hace improbable que la relación sonido-significado que implica esté sujeta a la arbitrariedad típica de las relaciones sonidosignificado que existen en la mayoría de entidades léxicas y gramaticales. Además el fenómeno estaría muy extendido para ser explicado mediante préstamo, descendencia de fuente lingüística común o azar. Concluye entonces Ohala que hay algo similar en todos los hablantes humanos, en todas las etapas de la historia, que hace que se usen $F 0$ (s) altos y/o elevaciones del $F 0$ para indicar preguntas, y $F 0$ (s) bajos y/o caídas del $F 0$ para indicar declaraciones.

\section{ESTUDIOS EN HUMANOS SOBRE LA PERCEPCIÓN TEMPRANA DE DECLARATIVOS E INTERROGATIVOS}

Sobre la percepción temprana de los contornos melódicos ascendentes y descendentes, en general, se sabe que un contorno melódico descendente (interpretado en un piano) puede ser procesado aun antes de nacer (en las semanas 35, 36 y 37 de gestación) y ser recordado por lo menos durante un mes después del nacimiento (Granier-Deferre, Bassereau, Ribeiro, Jacquet \& DeCasper, 2011). Se ha encontrado además que los recién nacidos prefieren los contornos melódicos ascendentes con respecto a los descendentes, y los tonos altos con respecto a los bajos (Frota et al., 2013), y que hacia las 68 horas de vida pueden discriminar entre distintos contornos tonales de palabras que no pertenecen a su lengua materna (Nazzi, Floccia \& Bertoncini, 1998).

Esta capacidad temprana para percibir contornos melódicos influye en la relación entre los bebés y sus cuidadores, pues se sabe que en el maternés se usan contornos melódicos ascendentes para llamar la atención del bebé o para darle turno en una interacción, y contornos melódicos descendentes para calmarlo o para finalizar la interacción (Español, 2007).

No obstante, al contrario de lo que podría pensarse, un estudio reciente reveló que las vocalizaciones de bebés de 10 y 14 meses son más frecuentes y largas en respuesta a las declaraciones de sus madres que en respuesta a las interrogaciones (Reimchen, 2013). Sin embargo, en el estudio también se encontró que los bebés de 10 y 14 meses vocalizan más (frecuencia y duración) en respuesta a las preguntas de sus madres cuando estas están definidas por contornos ascendentes de tono que cuando lo están por contornos descendentes; pero no sucede lo mismo con las declaraciones.

También influye la percepción temprana de la entonación en la conducta del bebé, pues el llanto de los recién nacidos (de entre 2 y 5 días de vida) presenta contornos tonales similares a los de la lengua hablada en su entorno (Mampe, Friederici, Christophe \& Wermke, 2009). Asimismo, se ha descubierto que hacia el primer año de vida las vocalizaciones de los bebés contienen frecuentemente contornos tonales en aumento que expresan peticiones e interés por objetos (Reimchen, 2013). 
En cuanto al estudio de la percepción de los contornos de entonación declarativo e interrogativo por parte de bebés, existen muy pocos reportes experimentales (al parecer básicamente cuatro). En el primero de ellos, los participantes fueron bebés de entre 6 y 8 y entre 10 y 12 meses de edad, hijos de familias monolingües estadounidenses (Best, Levitt $\&$ McRoberts, 1991). Mediante un método basado en el paradigma de habituación se estudió la capacidad de los bebés para discriminar entre oraciones declarativas $(D)$ y oraciones interrogativas $(I)$, en inglés y en español ${ }^{3}$. Las oraciones fueron producidas por una hablante nativa estadounidense y una mexicana, respectivamente. Los resultados indicaron que los bebés más pequeños $(6-8$ meses) diferenciaron las preguntas de las declaraciones en el idioma materno y en el desconocido, mientras que los mayores (10 - 12) no lo hicieron en ninguna lengua.

Los resultados de este estudio no son concluyentes por diversas razones:

1.- Mientras las interrogaciones en español tenían consistentemente un aumento final de entonación, las interrogaciones en inglés eran preguntas-wh (wh-questions), con una entonación final en descenso.

2.- Las declaraciones en ambos idiomas eran exclamativas (por ejemplo: - What a beautiful baby!; - ¡Qué niñita más linda!), $\mathrm{y}$ esto podría haber modificado su contorno canónico de entonación.

3.- Los estímulos constituyentes de las $I$ y las $D$ eran distintos (aparte de la ento-

3 Los métodos basados en el paradigma de habituación incluyen típicamente una fase de familiarización con un cierto estímulo (o tipo de estímulos), seguida de una fase de prueba en la que se presenta un estímulo (o tipo de estímulos) novedoso; la respuesta diferenciada ante los estímulos novedosos respecto a los presentados en la fase de familiarización se entiende como evidencia de la capacidad para discriminarlos o de la preferencia por unos de ellos (Doupe \& Kuhl, 1999). nación) en el orden de las palabras (por la inversión en las preguntas-wh, con el verbo auxiliar antepuesto al sujeto) y en las propiedades léxicas (presencia de la palabra-wh en las preguntas). Esto impide saber qué fue lo que realmente permitió o impidió la discriminación (concuerdan Frota et al., 2013).

4.- Afirman los autores del trabajo (Best et al., 1991) que el habla usada para dirigirse a los bebés no era apropiada para los participantes mayores del estudio (1012 meses), pues el habla dirigida a bebés de cerca de un año se refiere más a objetos y personas, mientras que la que se usa con bebés más jóvenes se refiere a estados y actividades del propio bebé (por lo visto esta última fue la utilizada en los experimentos).

En el segundo estudio, los participantes fueron bebés de 14 meses de edad promedio (en un rango de 4 a 24 meses), hijos de familias angloparlantes y se utilizó el método de habituación a una pantalla individual (basado en el paradigma de habituación) (Soderstrom et al., 2011). En este trabajo se puso a prueba la capacidad de los bebés para discriminar entre oraciones declarativas $(D)$ (terminadas en $F 0(\mathrm{~s})$ variables, como plano, caída y campana) y oraciones interrogativas (I) (terminadas en un aumento del $F 0$ ); ambas producidas por una hablante nativa, en inglés, y dirigidas al bebé, y ambas modificadas con un filtro de bajo nivel a $400 \mathrm{~Hz}^{4}$. Las $I$ no estaban invertidas y estaban constituidas por las mismas palabras que las $D$, de

4 El paso por el filtro de bajo nivel se usa como una manera de conservar los aspectos prosódicos de la señal del habla (acento, entonación y ritmo), permitiendo que sean percibidos, y quitar o atenuar otros (estructura fonética y fonotáctica) de manera que no se puedan distinguir las palabras (Burnham, Kitamura \& Vollmer-Conna, 2002). 
tal forma que lo único que diferenciaba los dos tipos de oraciones era la entonación ${ }^{5}$.

Los datos resultantes de este estudio no fueron suficientes para asegurar que los bebés pueden diferenciar las $D$ de las $I$. Sin embargo, en todos los participantes se evidenció una preferencia hacia las $I$ en la fase de familiarización, lo cual implica que atendieron a las características prosódicas / acústicas que diferenciaban los dos tipos de entonación. Estiman en consecuencia los autores del trabajo que los bebés tienen las habilidades perceptuales y atencionales necesarias para empezar a formar las categorías de declaracióninterrogación con anterioridad al conocimiento morfosintáctico, y que existe un sesgo categórico de aparición temprana hacia las preguntas o hacia características acústicas como la entonación en aumento (Soderstrom et al., 2011).

El tercer estudio fue realizado con bebés de 7 meses promedio de edad, hijos de familias angloparlantes, usando el método de preferencia de giro de cabeza (basado también en el paradigma de habituación) (Geffen \& Mintz, 2011). Se puso a prueba la capacidad de los bebés para diferenciar entre oraciones declarativas $(D)$ e interrogativas $(I)$, en inglés, que no solo diferían en sus contornos de en-

5 En el idioma inglés se pueden dar interrogaciones sin la inversión sintáctica de la oración, solamente marcadas por la entonación, en cuyo caso lo único que diferencia la declaración de la interrogación es la entonación (por ejemplo: - He bought bread at the market; - He bought bread at the market?), pero en este caso la interrogación no es una simple petición de información (como sí lo es - Did he buy bread at the market?), sino que además expresa sorpresa. En cambio en español, aunque cambios en el orden de las palabras pueden indicar una pregunta (p. ej. - ¿Compró él pan en el mercado?), la sola entonación interrogativa en una oración, que tiene las mismas palabras que una oración declarativa, expresa simplemente una petición de información (por ejemplo: - Él compró pan en el mercado; - ¿Él compró pan en el mercado?) (Face, 2005). tonación sino que además se diferenciaban en cuanto al auxiliar y a la inversión sintáctica de las $I$, y en cuanto a las palabras que las constituían (es decir, las oraciones $D$ e $I$ no compartían las mismas palabras, como en el estudio de Soderstrom et al., 2011). Todas las oraciones fueron proferidas por una mujer en el registro de habla dirigida al bebé.

Al igual que los resultados de un estudio no concluyente hecho con anterioridad por los mismos autores (Geffen, 2010), los resultados de este estudio indicaron que los bebés de 7 meses tienen la capacidad de diferenciar entre las $I$ y las $D$. Sin embargo, Geffen y Mintz (2011) aceptan que el orden de las palabras (inversión del sujeto) y la presencia del auxiliar $d o$ en las $I$ pudo influir en la discriminación, pero afirman que es improbable que los bebés de 7 meses representen los enunciados que escuchan de manera tal que esa información pueda ser relevante. Acerca de la diferencia de estos resultados con los del estudio de Soderstrom y colaboradores (2011), afirman Geffen y Mintz que en el procedimiento de Soderstrom el uso de preguntas no invertidas pudo haber alterado su entonación canónica; además señalan que el rango de edad de los participantes (4 a 24 meses) fue demasiado amplio.

El cuarto estudio se realizó con bebés de entre 5 y 6 y entre 8 y 9 meses de edad, hijos de familias monolingües hablantes del portugués (Frota et al., 2013). Valiéndose de una modificación del método de medición de la fijación visual (basado también en el paradigma de habituación) se estudió la capacidad de los bebés para discriminar entre oraciones interrogativas (que se responden con sí o no) $(I)$ y oraciones declarativas $(D)$, del portugués europeo estándar (en este idioma la diferencia entre $I$ y $D$ se indica solo por medios prosódicos). Tanto las $I$ como las $D$ fueron oraciones de una sola palabra (inventada) proferidas por una mujer, en el registro de habla dirigida al bebé. Los resultados revelaron que los bebés pueden discriminar el contraste de entonación de las $I$ con respecto al de las $D$ ya a los 5 meses, y que mantienen esta capacidad por lo menos hasta los 9 meses. Ya que los estímulos eran palabras sin sentido con estruc- 
turas fonológicas equivalentes, se entiende que la discriminación se realizó solamente a partir de las diferencias de entonación.

\section{ESTUdiOS EN ANIMALES NO HUMANOS SOBRE LA PERCEPCIÓN DE DECLARATIVOS E INTERROGATIVOS}

Con respecto al estudio en modelos animales de la percepción de los contornos de entonación declarativo e interrogativo, no parecen existir en la actualidad trabajos empíricos que permitan indicar si la capacidad de discriminar entre preguntas y declaraciones, solamente a partir del contorno de entonación, es una capacidad exclusivamente humana o es compartida en alguna medida con otras especies. Sin embargo, un estudio piloto realizado en Bogotá con ratas Wistar y con una metodología de reforzamiento operante diferencial, aplicada a una tarea de decisión en un laberinto en $\mathrm{T}$, indicó que estos roedores no tienen la capacidad de discriminar entre oraciones en español idénticas en todo sentido, salvo en su contorno de entonación (declarativas o interrogativas) (Barón, 2012). De cualquier manera, los datos de esta aproximación experimental al problema no son concluyentes.

\section{EPÍLOGO Y CONCLUSIONES}

Con el fin de justificar teóricamente el estudio comparado de la percepción de los contornos de entonación interrogativo y declarativo en humanos y otros animales se han presentado los siguientes argumentos:

1.- El enfoque de estudio psicolingüístico comparado es una forma fructífera de profundizar el conocimiento sobre la facultad humana del lenguaje.

2.- Una de las variables experimentales que se considera en ese enfoque es la isocronía, la cual parece ser un universal lingüístico relacionado con la percepción.

3.- Al igual que la isocronía, los contornos de entonación de las oraciones declarativas y de las oraciones interrogativas (en particular las que se responden con $s i$ o no) presentan características compartidas en alto grado en la diversidad de lenguas naturales.

Ahora bien, a pesar de que existen numerosas investigaciones en percepción del lenguaje en general en recién nacidos, en percepción de la entonación declarativa e interrogativa en adultos y en Psicología Comparada del lenguaje, los trabajos con bebés en cuanto a la percepción de la entonación declarativa e interrogativa son muy escasos y los estudios sobre este mismo tema en modelos animales son al parecer inexistentes.

En este sentido parece sensato estudiar en particular la percepción de declarativos e interrogativos, pues se entiende que las declaraciones y las preguntas son tipos básicos de oraciones y por lo tanto la habilidad para distinguirlas es crucial para procesar los demás tipos de oraciones. Además, la discriminación temprana entre la entonación declarativa y la interrogativa podría facilitar la adquisición de las estructuras sintácticas del lenguaje. En cuanto al orden pragmático, las preguntas (y sus respectivas respuestas) son cruciales para los procesos de interacción social y de comunicación (Frota et al., 2013; Geffen \& Mintz, 2011).

Estudiar la entonación declarativa e interrogativa específicamente en recién nacidos permite aclarar asuntos relacionados con las características ontogenéticas del desarrollo del lenguaje y con el involucramiento de aspectos innatos y de instrucción en la adquisición lingüística, como la necesidad de conocer la forma sintáctica de una pregunta y su relación con el papel de las interrogativas en el orden pragmático, o la relación de la forma fonológica de la pregunta y su estructura sintáctica. Además puede brindar información sobre el papel de mecanismos generales o de dominio específico en el tratamiento de la señal del habla, así como sobre la evolución filogenética del lenguaje y su relación con el simbolismo sonoro expresivo que se encuentra en animales no humanos.

Asimismo, la capacidad temprana para distinguir preguntas de declaraciones puede 
ayudar a quien aprende a hablar a descubrir ciertas distinciones pragmáticas antes de tener conocimiento léxico. Por ejemplo, la entonación de pregunta indica que se espera una respuesta de quien escucha, mientras que la de declaración indica un comentario dirigido hacia quien escucha (Best et al., 1991).

No obstante, el número de estudios sobre la entonación declarativa e interrogativa en bebés es reducido y solo han sido realizados con respecto a la percepción de tres idiomas (inglés, portugués europeo y español). Igualmente, en tres de cuatro casos se evaluó la percepción del idioma hablado en el contexto del sujeto, y en todos los estudios se utilizó alguna variante del paradigma de habituación y los estímulos se grabaron en el registro de habla dirigida al bebé.

Este último asunto podría resultar de particular importancia, pues se sabe que en el habla dirigida al bebé se modifican automática e invariablemente las características de la señal mediante la elevación general y la mayor variación del $F 0$, la exageración de los contornos de entonación y la articulación desmesurada de las vocales (respecto al registro ordinario del habla) (Burnham, Kitamura \& Vollmer-Conna, 2002). De esta forma se logra mantener la atención de los bebés con un registro que además se caracteriza por tener una alta proporción de oraciones interrogativas (Frota et al., 2013; Soderstrom et al., 2011). Por otra parte, como en el caso del estudio de Best y colaboradores (1991), un tipo particular de habla dirigida al bebé podría no ser el más adecuado para el rango de edad de un determinado estudio.

Resulta entonces conveniente incrementar el número de estudios sobre la percepción de interrogativos y declarativos en recién nacidos, modificando variables clave como el registro de la señal, el orden de las palabras y el uso de auxiliares (como do), la longitud de los enunciados, las edades de los participantes y el método experimental. Además, aparte de las técnicas basadas en el paradigma de habituación, existen diversas formas de estudiar la percepción temprana del habla en recién nacidos, e incluso en fetos, como el reforzamiento diferencial (DeCasper \& Spence,
1986), el procedimiento de giro de cabeza condicionado (Werker, Polka \& Pegg, 1997) y el paradigma de no coincidencia estándar desviado (Ortiz-Mantilla, Hamalainen \& Benasich, 2012), entre otros.

En cuanto al estudio de la percepción de los contornos de entonación declarativo e interrogativo en modelos animales, que de acuerdo con la revisión realizada para el trabajo que se informa no se ha realizado (salvo el estudio piloto que se mencionó), en principio puede referirse a la capacidad para diferenciar dichos contornos en varios idiomas, proferidos por distintos hablantes (hombres y mujeres) y con oraciones de distinta longitud. Puede utilizarse el filtro de bajo nivel para enfatizar la prosodia, si se encuentra conveniente, o utilizar exactamente las mismas palabras para expresar tanto los enunciados declarativos como los interrogativos, modificando tan solo el contorno de entonación, como es de uso común en el idioma español. Para este fin pueden utilizarse incluso los mismos estímulos que se han usado en los estudios con bebés sobre la percepción de las preguntas y las declaraciones (o modificaciones de estos).

En cuanto a los métodos experimentales relevantes puede usarse en el caso de los monos titíes el método de respuesta de orientación de la cabeza hacia el altoparlante (head orientation response toward the loudspeaker) (Ramus, Hauser, Miller, Morris \& Mehler 2000) o el muy similar procedimiento de familiarización-prueba (familiarization - test procedure) (Saffran et al., 2008). Con las aves puede usarse una técnica utilizada con pericos conocida como programa operante estándar de automoldeamiento (standard operant autoshaping program) (Dent, Brittan-Powell, Dooling \& Pierce, 1997), o el paradigma Go / NoGo, que ha sido utilizado con diamantes mandarín (Ohms, Gill, Heijningen, Beckers \& ten Cate, 2010). Con los roedores puede usarse un procedimiento de reforzamiento diferencial en cámara de condicionamiento operante, como el aplicado por Toro y Trobalon (2005), o el aplicado por Reed y colaboradores (2003). 
Encontrar que otros animales distintos al hombre tienen la capacidad para distinguir entre contornos de entonación declarativos e interrogativos a partir solamente de características prosódicas proporcionaría evidencia empírica sobre la hipótesis auditiva y la hipótesis universal fuerte, y se constituiría como evidencia en contra de la SiS pero no necesariamente en contra de la hipótesis de tono nuclear (pues esta última asume que existen tonos similares en todas las lenguas, pero postula que la asociación de estos con los significados es arbitraria, lo cual podría deberse a un tipo de adaptación específicamente humana).

Con respecto al estudio en general en modelos animales y en humanos, la modificación digital de enunciados interrogativos y declarativos puede permitir poner a prueba la existencia de un fenómeno similar al efecto de frontera de fonema, pero no referido a un espectro del habla entre fonemas sino a una suerte de espectro entre contornos de entonación (sobre el efecto de frontera de fonema véase Kuhl \& Padden, 1982). De esta manera podría resultar que existe también un límite tonal en el continuo entre un contorno declarativo y uno interrogativo, y que la discriminación entre dos muestras en este continuo se facilita si ambas están en extremos opuestos del espectro y se dificulta si están en el mismo extremo.

De la misma forma, podría existir algún tipo de efecto de magneto perceptual referido a los contornos de entonación, de manera que la generalización a partir de tipos ideales de contornos (prototipo) sea más amplia con respecto a la generalización a partir de tipos que no son el prototipo de la clase (sobre el efecto de magneto perceptual véase Kuhl, 1991). De existir un fenómeno con estas características habría que averiguar si al igual que el efecto de magneto perceptual es privativo de los humanos o si es compartido entre especies.

En síntesis, aumentar el número de estudios de la percepción de los contornos de entonación declarativo e interrogativo en recién nacidos (modificando variables clave) y realizar estudios sobre ese mismo tipo de per- cepción en modelos animales, como ratas, aves y monos, resulta viable y pertinente, $\mathrm{y}$ permitiría profundizar el conocimiento sobre las condiciones del lenguaje que son privativas de los hombres y las que son compartidas con otras especies, sobre las relaciones de forma y función en el componente fonológico de las lenguas del mundo, y sobre los universales y los precursores lingüísticos.

\section{REFERENCIAS BIBLIOGRÁFICAS}

Alarcos, E. (2000). Gramática de la lengua española [Spanish language grammar]. Madrid: Espasa Calpe.

Barón, L. (2012). Discriminación de entonación afirmativa e interrogativa en español por parte de ratas [Discrimination of interrogative and declarative intonation contours by rats]. En N. Cervone (Pres.), IV Congreso Internacional de Investigación y Práctica Profesional en Psicología (pp. 83-84). Buenos Aires: Argentina.

Barón, L. (2014). La psicolingüistica comparada: Investigación del lenguaje en humanos y animales desde el punto de vista cognitivo [The comparative psycholinguistics: Research on language in human and animals from the cognitive standpoint]. Manuscrito enviado para su publicación.

Berwick, R. \& Chomsky, N. (2011). The biolinguistic program: The current state of its evolution and development. En A. di Sciullo \& C. Boeckx (Eds.), The biolinguistic enterprise: New perspectives on the evolution and nature of the human language faculty (pp. 19-41). Oxford: Oxford University Press.

Berwick, R., Friederici, A., Chomsky, N. \& Bolhuis, J. (2013). Evolution, brain, and the nature of language. Trends in Cognitive Sciences, 17(2), 89-98. http://dx.doi.org/10.101 6/j.tics.2012.12.002 
Best, C., Levitt, A. \& McRoberts, G. (1991). Examination of language-specific influences in infants' discrimination of prosodic categories. Proceedings of the XIIth International Congress of Phonetic Sciences, Vol. 4 (pp. 162165). Aix-en Provence: Université de Provence, France.

Brainard, M. \& Doupe, A. (2002). What songbirds teach us about learning. Nature, 417(6886), 351-358. http://dx.doi.org/10.1038/417351a

Burnham, D., Kitamura, C. \& Vollmer-Conna, U. (2002). What's new, pussycat? On talking to babies and animals. Science, 296, 1435. http:// dx.doi.org/10.1126/science.1069587

Chomsky, N. (1997). Language and problems of knowledge. Teorema, 16(2), 5-33.

Chomsky, N. (2010). Some simple evo devo theses. How true might they be for language? En R. Larson, V. Deprez \& H. Yamakido (Eds.), The evolution of human language (pp. 45-62). Cambridge: Cambridge University Press. http:// dx.doi.org/10.1017/CBO9780511817755.003

Chomsky, N. (2011). Language and other cognitive systems. What is special about language? Language Learning and Development, 7, 263278. http://dx.doi.org/10.1080/15475441.201 1.584041

Cruttenden, A. (1981). Falls and rises: Meanings and universals. Journal of Linguistics, 17(1), 77-91. http://dx.doi.org/10.1017/S0022226700 006782

DeCasper, A. \& Spence, M. (1986). Prenatal maternal speech influences newborns' perception of speech sounds. Infant Behavior and Development, 9, 133-150. http://dx.doi.org/ 10.1016/0163-6383(86)90025-1

Dent, M., Brittan-Powell, E., Dooling, R. \& Pierce, A. (1997). Perception of synthetic /ba/-/wa/ speech continuum by budgerigars (Melopsittacus undulatus). Journal of Acoustical Society of America, 102(3), 1891-1897. http://dx.doi. org/10.1121/1.420111

Di Sciullo, A. et al. (2010). The biological nature of human language. Biolinguistics, 4, 1-31.
Doupe, A. \& Kuhl, P. (1999). Birdsong and human speech: Common themes and mechanisms. Annual Review of Neuroscience, 22, 567-631. http://dx.doi.org/10.1146/annurev.neuro.22.1.5 67

Elgier, A. \& Bentosela, M. (2009). El gesto de señalar: Una llave para la comunicación entre especies [The pointing gesture: A key for communication between species]. Interdisciplinaria, 26(2), 157-182.

Endress, A., Cahill, D., Block, S., Watumull, J. \& Hauser, M. (2009). Evidence of an evolutionary precursor to human language affixation in a non-human primate. Biology Letters, 5, 749-751. http://dx.doi.org/10.1098/rsbl.2009. 0445

Español, S. (2007). Lenguaje, comunicación e intersubjetividad: Una aproximación desde la psicología del desarrollo [Language, communication and intersubjectivity: A developmental psychology approach]. Subjetividad y Procesos Cognitivos, 10, 13-28.

Face, T. (2005). F0 peak height and the perception of sentence type in Castilian Spanish. Revista Internacional de Lingüistica Iberoamericana, 2(6), 49-65.

Frota, S., Butler, J. \& Vigário, M. (2013), Infants' perception of intonation: Is it a statement or a question? Infancy, 19(2), 194-213. http://dx. doi.org/10.1111/infa.12037

Geffen, S. (2010). Can infants discriminate between declaratives and interrogatives? Tesis de Maestría no publicada. University of Southern California, Los Angeles.

Geffen, S. \& Mintz, T. (2011, noviembre). Sevenmonth-olds discrimination of statements and questions. Trabajo presentado en la 36th Boston University Conference on Language Development, Boston, USA.

Gervain, J. \& Mehler, J. (2010). Speech perception and language acquisition in the first year of life. Annual Review of Psychology, 61, 191-218. http://dx.doi.org/10.1146/annurev.psych.09300 8.100408 
Ghazanfar, A. \& Maier, J. (2009). Rhesus monkeys (Macaca mulatta) hear rising frequency sounds as looming. Behavioral Neuroscience, 123(4), 822-827. http://dx.doi.org/10.1037/a0016391

Granier-Deferre, C., Bassereau, S., Ribeiro, A., Jacquet, A. \& DeCasper, A. (2011). A melodic contour repeatedly experienced by human near-term fetuses elicits a profound cardiac reaction one month after birth. PLoS ONE, 6(2), 1-10. http://dx.doi.org/10.1371/journal. pone.0017304

Gussenhoven, C. \& Chen, A. (2000). Universal and language-specific effects in the perception of question intonation. International Conference on the Processing of Spoken Language, 6(2), 14.

Hauser, M., Chomsky, N. \& Fitch, T. (2002). The faculty of language: What is it, who has it, and how did it evolve? Science, 298, 1569-1579. http://dx.doi.org/10.1126/science.298.5598.15 69

Heimbauer, A. (2012). Investigating speech perception in evolutionary perspective: Comparisons of chimpanzee (Pan troglodytes) and human capabilities. Tesis Doctoral no publicada. Georgia State University, Georgia.

Hirst, D. \& Di Cristo, A. (1998). A survey of intonation systems. En D. Hirst \& A. Di Cristo (Eds.), Intonation systems (pp. 1-44). Cambridge: Cambridge University Press.

Jakovcevic, A. \& Bentosela, M. (2012). Mira lo que te muestro: ¿Comunicación referencial entre perros domésticos (canis familiaris) y humanos? [Look what I show you: Referential communication between domestic dogs (canis familiaris) and humans?]. Interdisciplinaria, 29(1), 5-22.

Kuhl, P. (1991). Human adults and human infants show a "perceptual magnet effect" for the prototypes of speech categories, monkeys do not. Perception and Psychophysics, 50(2), 93107. http://dx.doi.org/10.3758/BF03212211

Kuhl, P. \& Padden, D. (1982). Enhanced discriminability at the phonetic boundaries for the voicing feature in macaques. Perception and Psychophysics, 32(6), 542-550. http://dx.doi. org/10.3758/BF03204208

Ladd, D. (1981). On intonational universals. En T. Myers, J. Laver \& J. Anderson (Eds.), The cognitive representation of speech (pp. 389397). Amsterdam: North-Holland Publishing. http://dx.doi.org/10.1016/S0166-4115(08)602 14-9

Lehiste, I. (1970). Suprasegmentals. Cambridge: Cambridge University Press.

Liberman, A. (1982). On finding that speech is special. American Psychologist, 37(2), 148167. http://dx.doi.org/10.1037//0003-066X.3 7.2.148

Lieberman, P. (1996). Some biological constraints on the analysis of prosody. En L. Morgan \& K. Demuth (Eds.), Signal to syntax: Bootstrapping from speech to grammar in early acquisition (pp. 55-66). Mahwah, NJ: Erlbaum Associates.

Longa, V. (2006). Sobre el significado del descubrimiento del gen FOXP2 [On the significance of the FOXP2 gene discovery]. Estudios de Lingüistica de la Universidad de Alicante, 20, 177-207.

Mampe, B., Friederici, A., Christophe, A. \& Wermke, K. (2009). Newborns' cry melody is shaped by their native language. Current Biology, 19, 1994-1997. http://dx.doi.org/10. 1016/j.cub.2009.09.064

Mehler, J., Christophe, A. \& Ramus, F. (2000). What we know about the initial state for language. En A. Marantz, Y. Miyashita \& W. O’Neil (Orgs.), Image, language, brain: Papers from the First Mind-Articulation Project Symposium (pp. 1-22). Cambridge, MA: MIT Press.

Morton, E. (1994). Sound symbolism and its role in non-human vertebrate communication. En L. Hinton, J. Nichols \& J. Ohala (Eds.), Sound symbolism (pp. 348-365). Cambridge: Cambridge University Press. http://dx.doi.org/10. 1017/CBO9780511751806.023 
Murphy, R., Mondragón, E. \& Murphy, V. (2008). Rule learning by rats. Science, 319, 1849-1851. http://dx.doi.org/10.1126/science.1151564

Nazzi, T., Floccia, C. \& Bertoncini, J. (1998). Discrimination of pitch contours by neonates. Infant Behavior and Development, 21(4), 779784. http://dx.doi.org/10.1016/S0163-6383(9 8)90044-3

Newport, E., Hauser, M., Spaepen, G., \& Aslin, R. (2004). Learning at a distance II. Statistical learning of non-adjacent dependencies in a non-human primate. Cognitive Psychology, 49, 85-117. http://dx.doi.org/10.1016/j.cog psy ch.2003.12.002

Ohala, J. (1984). An ethological perspective on common cross-language utilization of $\mathrm{F} 0$ in voice. Phonetica, 41, 1-16.

Ohms, V., Escudero, P., Lammers, K. \& ten Cate, C. (2012). Zebra finches and Dutch adults exhibit the same cue weighting bias in vowel perception. Animal Cognition, 15(2), 155-161. http://dx.doi.org/10.1007/s10071-011-0441-2

Ohms, V., Gill, A., Heijningen, C., Beckers, G. \& ten Cate, C. (2010). Zebra finches exhibit speaker-independent phonetic perception of human speech. Proceedings of the Royal Society B, 277, 1003-1009.

Ortiz-Mantilla, S., Hamalainen, J. \& Benasich, A. (2012). Time course of ERP generators to syllables in infants: A source localization study using age-appropriate brain templates. Neuroimage, 59(4), 3275-3287. http://dx.doi.org/10. 1016/j.neuroimage.2011.11.048

Peña, M., Pittaluga, E. \& Mehler, J. (2010). Language acquisition in premature and full-term infants. Proceedings of the National Academy of Sciences of the United States of America, 107, 3823-3828.

Pilley, J. \& Reid, A. (2011). Border collie comprehends object names as verbal referents. Behavioural Processes, 86(2), 184-195. http:// dx.doi.org/10.1016/j.beproc.2010.11.007

Pinker, S. \& Jackendoff, R. (2005). The faculty of language: what's special about it? Cognition,
95, 201-236. http://dx.doi.org/10.1016/j.cogn ition.2004.08.004

Ramus, F. (1999). Rythme des langues et acquisition du langage [Rhythm of language and language acquisition]. Tesis Doctoral no publicada. EHESS. Paris.

Ramus, F., Dupoux, E. \& Mehler, J. (2003). The psychological reality of rhythm classes: Perceptual studies. Trabajo presentado en el 15th International Congress of Phonetic Sciences, Barcelona, España.

Ramus, F., Hauser, M., Miller, C., Morris, D. \& Mehler, J. (2000). Language discrimination by human newborns and by cotton-top tamarin monkeys. Science, 288, 349-351. http://dx.doi. org/10.1126/science.288.5464.349

Ramus, F., Nespor, M. \& Mehler, J. (1999). Correlates of linguistic rhythm in the speech signal. Cognition, 73, 265-292. http://dx.doi. org/10.1016/S0010-0277(99)00058-X

Reed, P., Howell, P., Sackin, S., Pizzimenti, L. \& Rosen, S. (2003). Speech perception in rats: Use of duration and rise time cues in labeling of affricate/fricative sounds. Journal of the Experimental Analysis of Behavior, 80, 205215. http://dx.doi.org/10.1901/jeab.2003.80205

Reimchen, M. (2013). Infant vocal responses to questions and declaratives in maternal speech. Tesis de Maestría no publicada. University of Manitoba, Winnipeg.

Saffran, J., Hauser, M., Seibel, R., Kapfhamer, J., Tsao, F. \& Cushman, F. (2008). Grammatical pattern learning by human infants and cottontop tamarin monkeys. Cognition, 107, 479500. http://dx.doi.org/10.1016 /j.cognition.20 07.10 .010

Sinnott, J. \& Gilmore, C. (2004). Perception of place-of-articulation information in natural speech by monkeys versus humans. Perception \& Psychophysics, 66(8), 1341-1350. http://dx. doi.org/10.3758/BF03195002

Soderstrom, M., Ko, E. \& Nevzorova, U. (2011). It's a question? Infants attend differently to 
yes/no questions and declaratives. Infant Behavior and Development, 34, 107-110. http:// dx.doi.org/10.1016/j.infbeh.2010.10.003

Toledo, G. (2009). Métricas rítmicas en tres dialectos Amper-Hispanoamérica [Rhythmic metrics in three Amper-Hispanoamérica dialects]. Ianua. Revista Philologica Romanica, 9, $1-21$.

Toro, J. \& Trobalon, J. (2005). Statistical computations over a speech stream in a rodent. Perception and Psychophysics, 67, 867-875. http://dx.doi.org/10.3758/BF03193539

Trout, J. (2001). The biological basis of speech: What to infer from talking to the animals. Psychological Review, 108(3), 523-549. http:// dx.doi.org/10.1037//0033-295X.108.3.523

Trout, J. (2003). Biological specializations for speech: What can the animals tell us? Current Directions in Psychological Science, 12(5), 155-159. http://dx.doi.org/10.1111/1467-8721.t 01-1-01251

Vaissière, J. (2005). Perception of intonation. En D. Pisoni \& R. Remez (Eds.), Handbook of speech perception (pp. 236-263). Oxford, Blackwell. http://dx.doi.org/10.1002/978047 0757024.ch10

Werker, J., Polka, L. \& Pegg, J. (1997). The conditioned head turn procedure as a method for testing infant speech perception. Early Development and Parenting, 6, 171-178. http:// dx.doi.org/10.1002/(SICI)1099-0917(19 9709/ 12)6:3/4\%3C171::AID-EDP156\% 3E3.3.CO; 2-8

Yang, X. \& Liang, J. (2012). Declarative and interrogative intonations by brain-damaged speakers of Uygur and Mandarin Chinese. En Q. Ma, H. Ding \& D. Hirst (Eds.), Proceedings of the Sixth International Conference on Speech Prosody (pp. 701-704). Shanghai, China.

Yuan, J., Shih, C. \& Kochanski, G. (2002). Comparison of declarative and interrogative intonation in Chinese. In B. Bel \& I. Marlien (Eds.), Proceedings of the First International Conference on Speech Prosody (pp. 711-714). Aix-en-Provence, France.

\footnotetext{
Grupo de Estudios en Ciencias del Comportamiento Fundación Universitaria Los Libertadores Bogotá - Colombia
}

Fecha de recepción: 1 de agosto de 2013 Fecha de aceptación: 9 de abril de 2014 